\title{
From the time-reversal mirror to the instantaneous time mirror
}

\author{
Mathias Fink ${ }^{\mathrm{a}}$ and Emmanuel Fort \\ Institut Langevin, ESPCI ParisTech, CNRS UMR 7587, PSL Research University, \\ 1 rue Jussieu, 75005 Paris, France
}

\begin{abstract}
Received 4 September 2016 / Received in final form 31 October 2016
\end{abstract} Published online 25 May 2017

\begin{abstract}
Because time and space play a similar role in wave propagation, wave control can be obtained by manipulating spatial boundaries or by manipulating time boundaries. These two dual approaches will be discussed in this paper in the context of the generation of time-reversed waves. The first approach uses the "time-reversal mirror" approach with wave manipulation along a spatial boundary sampled by a finite number of antennas. In the second approach, waves are manipulated from a time boundary and we show that "instantaneous time mirrors", simultaneously acting in the entire space can also radiate time-reversed waves.
\end{abstract}

\section{Introduction}

It is well known that, although classical mechanics equations are reversible on the microscopic scale, it is difficult to test the time reversal invariance of a complex system of particles. It requires introducing a "mirror" of the time variable that can be ascribed to the sudden change in initial conditions of the complex system resulting in full motion reversal. It was first the Austrian physicist J. Loschmidt that challenged Boltzmann's attempt to describe irreversible macroscopic processes with reversible microscopic equations that imagined daemons capable of inversing instantaneously all velocities of all particles in a gas. The daemons have to prepare the system in a new initial condition where all the velocities are reversed. However such a proposal remains mainly a thought experiment, the extreme sensitivity to initial conditions that lies at the heart of chaotic phenomena in nonlinear dynamics renders any such particulate scheme impossible to realize.

Time-reversal invariance does not occur only in classical mechanics, it also occurs in wave physics to the extent that waves propagate without any dissipative process. Waves are more amenable to time-reversal schemes since they can be described in many situations by a linear operator and any error in the initial conditions will not suffer from chaotic behavior. It is interesting to note that both the holographic principle and the time-reversal mirror approach are mainly based on the time-reversal invariance of wave equations. They also rely on the fact that any wave field can be completely determined within a volume knowing the field (and its normal derivative)

\footnotetext{
${ }^{a}$ e-mail: mathias.fink@espci.fr
} 
on any enclosing surface $[1,2]$. Hence, information reaching the $2 \mathrm{D}$ surface is sufficient to recover all the fields inside the whole volume. Based on these properties, Denis Gabor [1] introduced the Holographic method providing an elegant way to backpropagate a monochromatic wave field towards its initial source and to obtain a $3 \mathrm{D}$ images of any radiating object. Time-reversal mirrors (TRMs) exploit also the same principles for broadband wavefield to physically create a time-reversed wave that exactly refocus back, in space and time, to the original source regardless of the complexity of the medium as if time were going backwards. This latter approach has been implemented with acoustic [3,4], electromagnetic [5] and water waves [6,7]. It requires the use of emitter-receptor antennas positioned on an arbitrary enclosing surface. The wave is recorded, digitized, stored, time-reversed and rebroadcasted by the same antenna array. If the array intercepts the entire forward wave with a good spatial sampling, it generates a perfect backward-propagating copy. Note that for optical waves, this processing is difficult to be implemented $[8,9]$ and the standard solution is to work with monochromatic light and to use nonlinear regimes such as three-wave of four-wave mixing $[10,11]$.

We have seen that the Loschmidt approach to design a "time machine" for particles is based on the sudden change in initial conditions of a complex system. The motion or "state" of an N particle system is determined at one time by the $\mathrm{N}$ positions and $\mathrm{N}$ velocities of the particles as a point in a $6 \mathrm{~N}$ phase space described by $\left\{\mathbf{r}_{i}, \mathbf{v}_{i}\right\}$. The Loschmidt daemons prepare new initial conditions for the $\mathrm{N}$ particles as $\left\{\mathbf{r}_{i},-\mathbf{v}_{i}\right\}$ by reversing the $\mathrm{N}$ velocities

In order to understand the way to design such a "time machine" for waves, we have to take into account the exact nature of the wave equation. It is a partial differential equation of order 2 and the wave field $\varphi(\mathbf{r}, t)$ is a continuous function of 4 variables (3 dimensions in space and one in time). Therefore the solution to the wave equation has to be a function described over a "hyper volume" with 4 variables and its boundary is a "hypersurface" with 3 variables [12].

In fact, there are two possibilities for the 3 variables of the hypersurface. It can be 2 spatial dimensions and one time dimension. This is the so called Cauchy Boundary Conditions (BCs) that prescribe both the field $\varphi(\mathbf{r}, t)$ and its normal derivative $\frac{\partial \varphi(\mathbf{r}, t)}{\partial n}$ for $\mathbf{r}$ along a $2 \mathrm{D}$ surface $\mathbf{S}$ for all time t. Once we know $\left\{\varphi(\mathbf{r} \in \mathbf{S}, t), \frac{\partial \varphi(\mathbf{r} \in \mathbf{S}, t)}{\partial n}\right\}$ we can use the Huygens-Fresnel-Helmholtz theorem to predict the field in all the hypervolume as

$$
\varphi(\mathbf{r}, t)=\int_{-\infty}^{t} d t^{\prime} \iint_{S}\left\{G\left(\mathbf{r}, \mathbf{r}^{\prime} ; t-t^{\prime}\right) \frac{\partial \varphi\left(\mathbf{r}^{\prime}, t^{\prime}\right)}{\partial n}-\frac{\partial G\left(\mathbf{r}, \mathbf{r}^{\prime} ; t-t^{\prime}\right)}{\partial n} \varphi\left(\mathbf{r}^{\prime}, t^{\prime}\right)\right\} d^{2} \mathbf{r}^{\prime}
$$

with $G\left(\mathbf{r}, \mathbf{r}_{0} ; t\right)$ the spatio-temporal Green's function solution of the wave equation with an impulsive point-like source located in $\mathbf{r}_{0}$.

The other possibility is a hypersurface described by 3 spatial dimensions where both the field $\varphi\left(\mathbf{r}, t_{m}\right)$ and its time derivative $\frac{\partial \varphi\left(\mathbf{r}, t_{m}\right)}{\partial t}$ for all $\mathbf{r}$ are prescribed at a given time $t_{m}$. This is the Cauchy initial Conditions (ICs) that are described by the wavefield state $\left\{\varphi, \frac{\partial \varphi(\mathbf{r}, t)}{\partial t}\right\}_{t_{m}}$. This is the initial value theorem that is classically called the Cauchy problem with the wave field at time $t$ and position $\mathbf{r}$ given by:

$$
\varphi(\mathbf{r}, t)=\iiint_{V}\left\{G\left(\mathbf{r}, \mathbf{r}^{\prime} ; t-t_{m}\right) \frac{\partial \varphi\left(\mathbf{r}^{\prime}, t_{m}\right)}{\partial t}-\frac{\partial G\left(\mathbf{r}, \mathbf{r}^{\prime} ; t-t_{m}\right)}{\partial t} \varphi\left(\mathbf{r}^{\prime}, t_{m}\right)\right\} d^{3} \mathbf{r}^{\prime}
$$

These two possibilities of Cauchy conditions (BCs) or (ICs) give rise to two dual approaches to create a "time machine" for waves. 
Section 2 is devoted to the "time-reversal mirror" (TRMs) concept that was initially developed and experimented with acoustic waves and later with microwaves. It refers to the manipulation of boundary conditions on a $2 \mathrm{D}$ surface sampled by a finite number of antennas.

Section 3 will be devoted to a second approach "à la Loschmidt" with wave control by manipulation of the initial conditions. It refers to the concept of "instantaneous time mirrors" (ITMs) that mimics the role of Loschmidt daemons, simultaneously acting in the entire space at once to radiate time-reversed waves, and we will show experimental evidence of this process with water waves.

\section{The time-reversal mirror approach "à la Huygens"}

We have seen that there are two dual approaches to predict a wavefield inside a hypervolume. We will now focus on the first choice where we used BCs instead of ICs. We know that if we record an incoming wavefield along a $2 \mathrm{D}$ surface during a sufficient time, the Huygens-Fresnel- Helmholtz-Kirchoff integral theorem allows recovering the wavefield at any time (past and future) in the whole volume.

Therefore a time-reversal experiment "à la Huygens" can be conceived in the following way. During a first step an initial source radiates a transient wavefield inside a volume surrounded by a $2 \mathrm{D}$ surface $\mathbf{S}$ along which the field and its normal derivative are recorded and stored as $\left\{\varphi(\mathbf{r} \in \mathbf{S}, t), \frac{\partial \varphi(\mathbf{r} \in \mathbf{S}, t)}{\partial t}\right\}$ during a sufficient amount of time to be sure that the incoming field as completely disappear along $\mathbf{S}$.

Employing a source term $s(\mathbf{r}, t)$ the radiated wavefield verifies the wave equation

$$
\Delta \varphi(\mathbf{r}, t)-\frac{1}{c(\mathbf{r})^{2}} \frac{\partial^{2} \varphi(\mathbf{r}, t)}{\partial t^{2}}=s(\mathbf{r}, t) .
$$

As we are in a causal universe, $\varphi(\mathbf{r}, t)$ is of course the causal solution of equation (3). Besides, due to the time reversal symmetry of the wave operator, there is also an anti-causal solution (the advanced one) that is never observed.

In the second step of the time-reversal experiment, our goal is to radiate from the boundary such an advanced solution. New boundary conditions have to be created along surface $\mathrm{S}$ with a wavefield oscillating in a time-reversed chronology compared to the chronology of the causal field. These time-reversed boundary conditions along $\mathrm{S}$ can be written $\left\{\varphi(\mathbf{r} \in \mathbf{S}, T-t), \frac{\partial \varphi(\mathbf{r} \in \mathbf{S}, T-t)}{\partial n}\right\}$ where $T$ is a causal delay needed to record and reemit the signals. Experimentally, it required the use of emitter-receptor antennas positioned on surface $\mathrm{S}$ that recorded, digitized, stored, time-reversed and rebroadcasted by the same antenna array both the field and its time derivative (see Fig. 1).

These new boundary conditions radiated a wave that is going backward to the source: the so-called time-reversed field $\varphi_{t r}(\mathbf{r}, t)$. It can be computed using the Helmholtz-Kirchhoff integral that is valid inside a zone without source (see Eq. (1)).

Instead of directly computing this integral, there is a straightforward way to predict the time-reversed field if we note that that this new wavefield verifies a homogeneous wave equation with the time-reversed BCs (the original source is no longer present)

$$
\Delta \varphi_{t r}(\mathbf{r}, t)-\frac{1}{c(\mathbf{r})^{2}} \frac{\partial^{2} \varphi_{t r}(\mathbf{r}, t)}{\partial t^{2}}=0 .
$$

Therefore, it cannot be equal to $\varphi(\mathbf{r}, T-t)$. In fact, it is not enough to time-reverse the wavefield on the boundary S. You also have to time-reverse the source term 


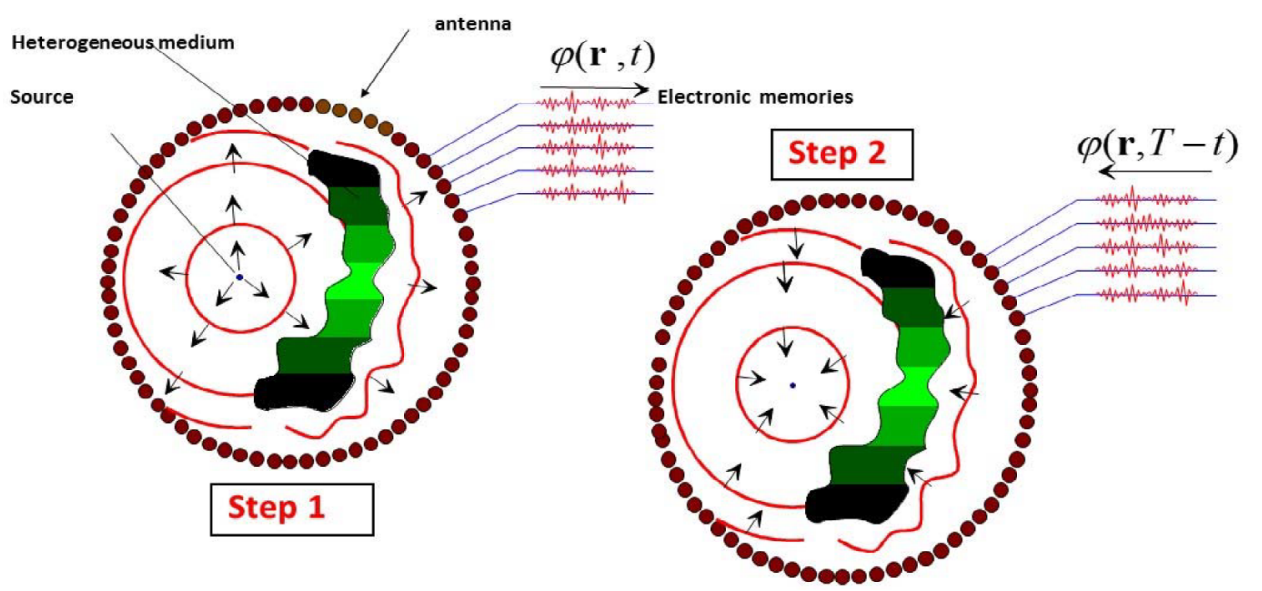

Fig. 1. Schematic of the time-reversal mirror. (a) Recording step: a closed surface is filled with transducer elements. A point like source generates a wave front which is distorted by heterogeneities. The distorted wavefield is recorded on the cavity elements. (b) Timereversed or reconstruction step. The recorded signals are time-reversed and reemitted by the elements. The time-reversed field back-propagates and refocuses exactly on the initial source.

on equation (3) and $\varphi(\mathbf{r}, T-t)$ indeed verifies the following equation deduced from equation (3) where $t$ is replaced by $T-t$

$$
\Delta \varphi(\mathbf{r}, T-t)-\frac{1}{c(\mathbf{r})^{2}} \frac{\partial^{2} \varphi(\mathbf{r}, T-t)}{\partial t^{2}}=s(\mathbf{r}, T-t) .
$$

This means that in order to achieve a perfect time reversal, both the source has to be transformed into a sink (the time-reversal of a sources $(\mathbf{r}, T-t)$ ), and also the field and its normal derivative on surface $S$ has to be time reversed. Note that if the source is impulsive, it writes $s(\mathbf{r}, t)=S(\mathbf{r}) \delta(t)$ and as $\delta(t)=\delta(-t)$ and we easily find that $[13]$

$$
\varphi_{t r}(\mathbf{r}, t)=\varphi(\mathbf{r}, T-t)-\varphi(\mathbf{r}, T+t) .
$$

This result means that to obtain the advanced wavefield, one has not only to timereverse the field but the original source as well. Equation (6) can be interpreted as the difference of advanced and retarded waves centered on the initial source position. The converging wave (advanced) collapses at the origin and is followed by a diverging (retarded) wave. Thus the time-reversed field observed as a function of time, shows two wavefronts of opposite sign. The wave re-emitted by the time reversal mirror looks like a convergent wavefield during a given period, but a wavefield doesn't know how to stop. When the converging wavefield reaches the location of the initial source location, it collapses and then continues its propagation as a diverging wavefield.

In the case of a punctual impulsive source term located at $\mathbf{r}_{0}$ we obtain for the time-reversed field:

$$
\varphi_{t r}(\mathbf{r}, t)=G\left(\mathbf{r}, \mathbf{r}_{0} ; T-t\right)-G\left(\mathbf{r}, \mathbf{r}_{0} ; T+t\right) .
$$

If the point-like source is monochromatic with pulsation $\omega$ we obtain through a Fourier transform a field pattern proportional to

$$
\hat{\phi}_{t r}(\mathbf{r}, \omega) \propto-2 j \operatorname{Im} \hat{G}\left(\mathbf{r}, \mathbf{r}_{0}, \omega\right)
$$




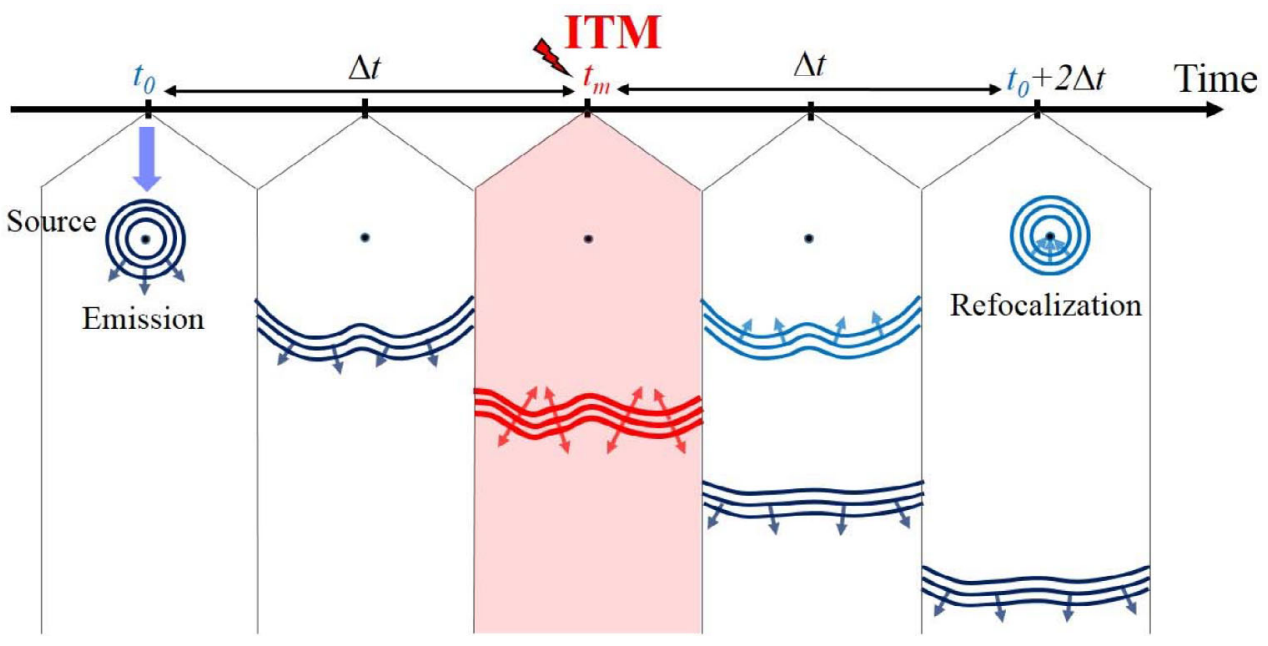

Fig. 2. Schematic of the Instantaneous Time Mirror. A wave source emits at time $t_{0}$ a wave packet which propagates in a given medium. A sudden spatially homogeneous disruption of the wave propagation properties occurs in the entire medium at time $t_{m}=t_{0}+\Delta t$. It results in the production of a counter propagating time-reversed wave in addition to the initial forward propagating wave. The counter propagating wave refocuses at the source position at time $t_{0}+2 \Delta t$.

with $\hat{G}\left(\mathbf{r}, \mathbf{r}_{0}, \omega\right)$ being the monochromatic Green's function. The time-reversed field is then proportional to the imaginary part of the Green's function and it is a universal result valid for any complex media provided that there is no dissipation. In free space, as the Green's function is a diverging spherical wave, the focal spot is limited to one half-wavelength as it is well known. Note that in complex media the field amplitude at the focal point is directly proportional to the LDOS, the so-called local density of states that depends on the medium complexity. Thus, the time-reversed field at the source point and at the focal time is directly proportional to the number of modes excited by the source.

To achieve a perfect time reversal both the field on the surface of the cavity has to be time reversed, and the source has to be transformed into a sink [14]. In this manner one may achieve time-reversed focusing below the diffraction limit. The role of the new source term in equation (5) is to transmit a diverging wave that exactly cancels the outgoing spherical wave.

In a monochromatic approach, taking into account the evanescent waves concept, the necessity of replacing a source by a sink in the complete time-reversed operation can be interpreted as follows. In the first step, a point-like source of size much smaller than a wavelength radiates a field that can be described as a superposition of homogeneous plane waves propagating in the various directions $\vec{k}$ and of decaying, nonpropagating, evanescent plane waves. The evanescent waves contain information on fine scale features of the source; they decay exponentially with distance and do not contribute to the far field. If the TRM is located in the far field of source, the time-reversed field retransmitted by the mirror does not contain these evanescent components. The role of the sink is to radiate exactly, with the good timing, the evanescent waves that have been lost during the first step. The resulting field contains the evanescent part that is needed to focus below diffraction limits. Time reversal below the diffraction limit has been experimentally demonstrated in acoustics, using 
an acoustic sink placed at the focal point. Focal spots of size $\lambda / 14$ have been observed by de Rosny et al [14]. One drawback is the need to use an active source at the focusing point to exactly cancel the usual diverging wave created during the focusing process.

Another solution is to create a passive sink that behaves as a perfect absorber of subwavelength size and it is concept that is currently studied by different teams in optics and in acoustics [15-17].

\section{The “instantaneous time mirror" mimicking Loschmidt for waves}

To mimic the Loschmidt approach in wave physics, one has first to measure an incoming wavefield at one specific time $t_{m}$ in the whole volume $\left\{\varphi, \frac{\partial \varphi}{\partial t}\right\}_{t_{m}}$. Then the analog for wave of the particle velocity reversal is to prepare a new set of initial conditions $\left\{\varphi,-\frac{\partial \varphi}{\partial t}\right\}_{t_{m}}$ where the sign of the time derivative has been reversed. Such an initial conditions will give rise to a time reversed wave. Even if this solution is appealing, it is not clear how to prepare such a new wave field pattern. It is in this context that an interesting solution was recently proposed [18] in the field of water waves. Because of the wave superposition principle, the emergence of this time reversed wave is however not limited to this choice of new initial conditions. For instance, a new initial condition $\{\varphi, 0\}_{t_{m}}$ can be split into $\frac{1}{2}\left\{\varphi, \frac{\partial \varphi(\mathbf{r}, t)}{\partial t}\right\}_{t_{m}}$ associated with a forward wave and $\frac{1}{2}\left\{\varphi,-\frac{\partial \varphi(\mathbf{r}, t)}{\partial t}\right\}_{t_{m}}$ associated with a backward time reversed wave. This particular disruption erases the arrow of time starting from a "frozen" picture of the wave field at time $t_{m}$ with no favored direction of propagation. Similarly, a new set of initial condition $\left\{0, \frac{\partial \varphi(\mathbf{r}, t)}{\partial t}\right\}_{t_{m}}$ in which the wave field is null would also comprise a backward propagating wave with negative sign. More generally, any superposition of the old initial conditions $\left\{\varphi, \frac{\partial \varphi}{\partial t}\right\}_{t_{m}}$ with a new set of initial conditions $\left\{0, f\left(\frac{\partial \varphi}{\partial t}\right)\right\}_{t_{m}}$ with $f$ being any function of $\frac{\partial \varphi}{\partial t}$ results in the superposition of a forward and backward propagating wave.

To prepare such a new wave pattern, we use the fact that a sudden modification of the wave celerity in the whole medium at time $t_{m}$ results in a new source term that depends of the incoming wavefield observed at time $t_{m}$. This offers a straightforward way to experimentally implement an instantaneous time reversal mirror. To understand the origin of this source term in the d'Alembert's wave equation, let us introduce a time-dependent phase velocity $c(t)=c_{0} / n(t)$, where $n(t)$ is a time-dependent index and $c_{0}$ is the initial phase velocity. The disruption undergone by the medium at time $t_{m}$ can be modelled by a $\delta$-Dirac function such that $c(t)^{2}=c_{0}^{2}\left(1+\alpha \delta\left(t-t_{m}\right)\right)$. The wave equation can be written as a nonhomogeneous equation in which the equivalent source term $s(\mathbf{r}, t)$ is induced by the velocity disruption:

$$
\Delta \varphi(\mathbf{r}, t)-\frac{1}{c_{0}^{2}} \frac{\partial^{2} \varphi(\mathbf{r}, t)}{\partial t^{2}}=s(\mathbf{r}, t)
$$

with

$$
s(\mathbf{r}, t)=-\frac{\alpha}{c_{0}^{2}} \delta\left(t-t_{m}\right) \frac{\partial^{2} \varphi(\mathbf{r}, t)}{\partial t^{2}} .
$$

The source term is localized in time but delocalized in space. It corresponds to an instantaneous source that is proportional to the second time derivative of the wave field at the instant $t_{m}$ of the disruption. This source term suddenly creates a set of real monopolar sources $s(\mathbf{r}, t)$, instantaneously in the whole space which isotropically radiate, generating in all directions an additional wave field. This instantaneous 
source term in the wave equation is equivalent to a change in the initial conditions (see Eq. (2)) that becomes now the superposition of the original state of the unperturbed wave field $\left\{\varphi, \frac{\partial \varphi}{\partial t}\right\}_{t_{m}}$ plus an added term $\left\{0, \frac{\alpha}{c_{0}^{2}} \frac{\partial^{2} \varphi}{\partial t^{2}}\right\}_{t_{m}}[12,18]$. This last term generates both a forward-propagating wave field and a time-reversed backwardpropagating wave field that are proportional to the time derivative of the original incident wave field.

It is in the field of gravity-capillary waves that such an experiment was recently described [18]. Since the surface wave celerity depends on the effective gravity $g$, the disruption of the celerity is simply achieved by applying a vertical impulsive acceleration to the whole liquid bath that change $g$ in $g+\gamma_{m}$. A bath of water is placed on a shaker to control its vertical motion. A tip is used to hit the liquid surface and generate a point-like source of water waves. Figure 3a shows a sequence of images of the wave propagation on the bath taken from above. A circular wave packet centered on the impact point is emitted as the tip hits the surface. Due to the dispersive nature of the gravity-capillary wave the wave profile is modified with time. The average wave propagation velocity is of the order of magnitude of $10 \mathrm{~cm} / \mathrm{s}$. After a time $t_{m}=60 \mathrm{~ms}$, a vertical downwards acceleration is applied to the bath. It reaches $\gamma_{m}=-18 \mathrm{~g}$ in approximately $2 \mathrm{~ms}$. Such an impulsive change of wave celerity can be described by a delta function in time. The propagation of the initial outwards propagating wave is not significantly affected by this disruption. However, at the time of disruption, we observe the radiation of a backwards converging circular wave packet which focus back to the initial source and that diverge again after. Such a behavior is analog to classical time-reversal mirrors except it is obtained without any antenna array or memory. The information stored in the whole medium at one instant plays the role of a bank of memories. We observed the emergence of the advanced Green's function from the bath and it is followed after the collapse by a diverging wave. This result can be extended to any type of source and Figure $3 \mathrm{~b}$ shows an impressive example with a metallic "smiley" hitting the liquid surface and radiating a complex field pattern. The ITM is activated at a time $t_{m}$ where the field structure has apparently completely loss the shape of the smiley and by virtue of the timereversal symmetry, one observe a backward wave that recreate a real image of the smiley in the bath !!!

It is also interesting to interpret this experiment in term of the two concepts of time refection and time refraction [18-21]. When we introduced an instantaneous time disruption for the wave speed or for the time-dependent index of refraction as $c(t)^{2}=c_{0}^{2}\left(1+\alpha \delta\left(t-t_{m}\right)\right)$, we have to remember that a Dirac pulse is the limit of a rectangular pulse with two time discontinuities. Therefore the instantaneous time disruption for the index can be considered as the limiting case of a rectangular time profile with two discontinuities. At time $t_{m}^{-}$, the wave speed jumps from $c_{0}$ to $c_{1}=c_{0} / n_{1}$ and then, at time $t_{m}^{+}$, changes back to its original value $c_{0}$. A temporal discontinuity in a homogeneous medium conserves the momentum but not the energy. In our experiment, this energy brought to the wave field is provided by the jolt. The time analog of the Fresnel formula can be obtained from conservation laws Hence, a monochromatic wave $e^{i\left(\mathbf{k} \cdot \mathbf{r}+\omega_{0} t\right)}$ of wave vector $k$ and angular frequency $\omega_{0}$ is split at the time discontinuity in a "transmitted" wave $t_{01} e^{i\left(\mathbf{k} \cdot \mathbf{r}+\omega_{1} t\right)}$ and a 'reflected' wave $r_{01} e^{i\left(\mathbf{k} \cdot \mathbf{r}+\omega_{1} t\right)}$, where $\omega_{1}=\omega / n_{1}$ is the angular frequency in medium 1 , and $t_{01}$ and $r_{01}$ are temporal Fresnel coefficients for time refraction and reflection, respectively. Each wave emerging from the first temporal discontinuity will be split again into two waves at the second discontinuity (see Fig. 4). This time slab is the time analogue of a Fabry-Pérot resonator. However, because of causality, multiple reflections are not permitted. The time-reversed wave field is thus the result of interference between two delayed backward waves with opposite signs (because $r_{01} t_{10}=-r_{10} t_{01}$ ). 


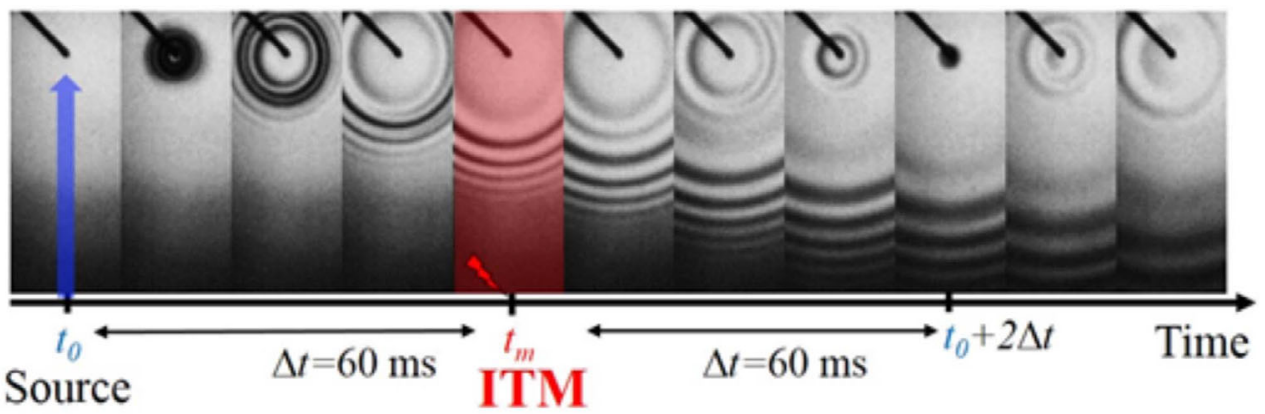

(a)

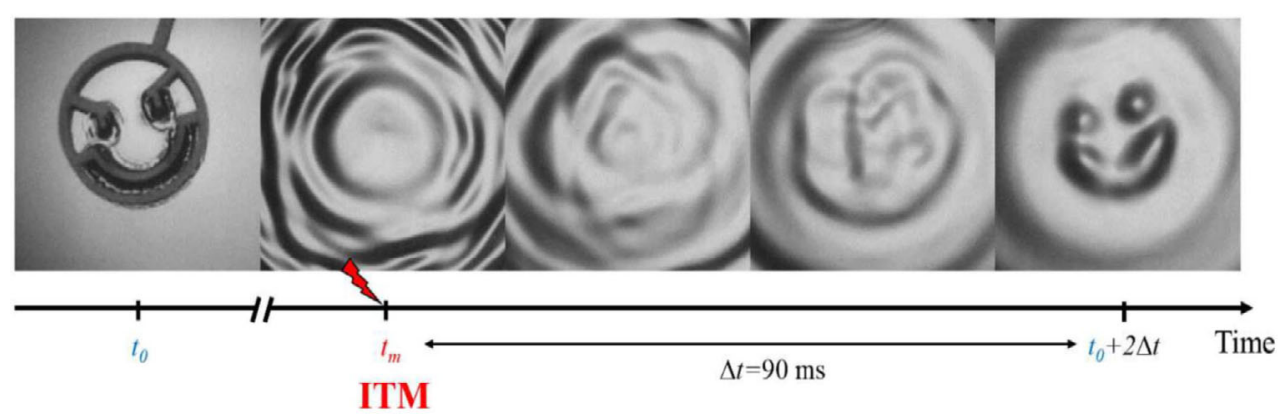

(b)

Fig. 3. (a) Water wave experiment. Image sequence of an ITM experiment with a point source showing the divergent wave and the time reversed wave which diverges again after focusing back at the source position. $\gamma_{m}=-2 l g$ and $\Delta t=60 \mathrm{~ms}$. (b) Image sequence of the water wave instantaneous time reversal of a complex wave field. The source is composed of an object that hits the water surface in the shape of a Smiley. The size of the images is approximately $8 \mathrm{~cm}$.

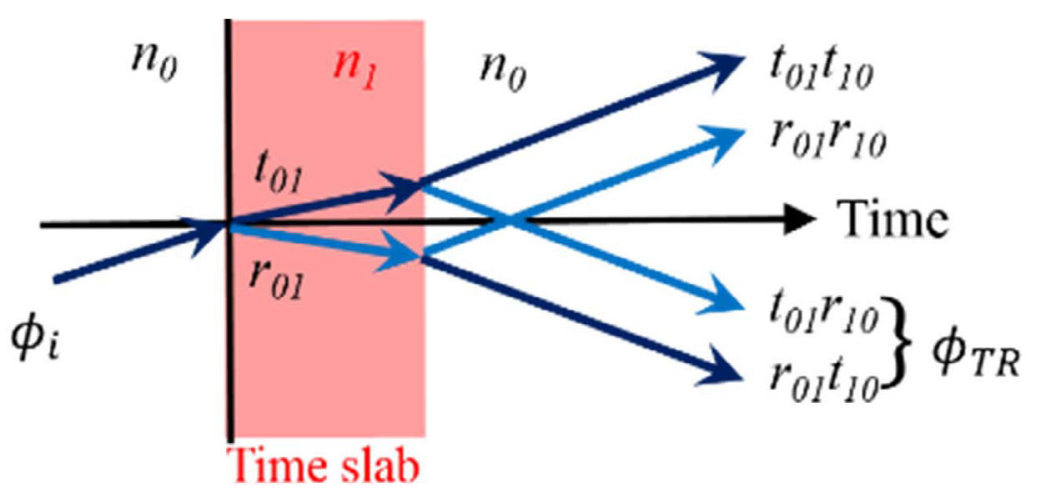

Fig. 4. Schematics of an incident wave field $\phi_{i}$ impinging on a time slab. $\phi_{T R}$ is the time reversed field. $t_{i j}$ and $r_{i j}$ are the transmission and reflection time Fresnel coefficients between medium $i$ and $j$. 
This explains why the resulting time-reversed field is not the perfect time reverse of the incident wave field $\varphi$, but rather of its derivative $\frac{\partial \varphi}{\partial t}$ in the limiting case of an instant disruption as the delayed between the two backward waves tends to zero.

For any source of complex shapes, these backwards waves recreated a perfect image of the initial source as in a holographic experiment, except that here the hologram is spread in the whole space and read by the action of the temporal discontinuity.

\section{Conclusion}

In this paper, we presented the two main approaches that can be used to generate time-reversed waves. The first approach uses "time-reversal mirrors" with wave manipulation along a spatial boundary sampled by a finite number of antennas. There are plenty applications of this approach for telecommunication, imaging, therapy and defense. In the second approach, we have shown that waves can also be manipulated on time boundaries by sudden modifications of the wave speed in the whole medium. Such an approach of "instantaneous time mirrors" mimics the Loschmidt point of view and is very efficient to radiated time-reversed waves without the use of any antenna.

The authors want to acknowledge the major influence of Roger Maynard on this research work. Roger Maynard was extremely interested by the arrow of time. Very often, he discussed with us the different ways to conduct time-reversal experiments. Shortly before he passed away, we shew him the movie illustrating the water wave instantaneous time reversal experiment with the smiley. We will never forget the pleasure he had discussing with us this experiment.

The authors thank also Vincent Bacot, Matthieu Labousse and Antonin Eddi for fruitful and stimulating discussions. They also acknowledge the support of the AXA research fund and LABEX WIFI (Laboratory of Excellence ANR-10-LABX-24) within the French Program "Investments for the Future" under reference ANR-10- IDEX-0001-02 PSL*.

\section{References}

1. D. Gabor, A new Microscopic Principle, Nature, 161, 777 (1948)

2. N. Bojarski, A survey of the near-field far-field inverse scattering inverse source integral equation, IEEE Trans. Antenna Prop. 30, 975 (1982)

3. M. Fink, Time reversal of ultrasonic fields i Basic principles, IEEE Trans. Ultrason. Ferroelectr. Freq. Control. 39, 555 (1992)

4. M. Fink, Time reversed acoustics, Phys. Today, 50, 34 (1997)

5. G. Lerosey, J. de Rosny, A. Tourin, M. Fink, Time reversal of electromagnetic waves, Phys. Rev. Lett. 92, 193904 (2004)

6. A. Przadka, et al., Time reversal of water waves, Phys. Rev. Lett. 109, 064501 (2012)

7. A. Chabchoub, M. Fink, Time-reversal generation of rogue waves, Phys. Rev. Lett. 112, $124101(2014)$

8. J. Aulbach, B. Gjonaj, P.M. Johnson, A.P. Mosk, A. Lagendijk, Control of light transmission through opaque scattering media in space and time, Phys. Rev. Lett. 106, 103901 (2011)

9. O. Katz, E. Small, Y. Bromberg, O. Silberberg, Focusing and compression of ultrashort pulses through scattering media, Nature Photon. 5, 372 (2011)

10. A. Yariv, Four wave nonlinear optical mixing as real time holography, Opt. Commun. 25, $23(1978)$

11. D.A.B. Miller, Time reversal of optical pulses by four-wave mixing, Opt. Lett. 5, 300 (1980) 
12. G. Barton, Elements of Green's Functions and Propagation: Potentials, Diffusion, and Waves (Oxford Science Publications, 1989)

13. D. Cassereau, M. Fink, Time-reversal of ultrasonic fields-Part III: Theory of the closed time reversal cavity, IEEE Trans. Ultrason. Ferroelec. Freq. Contr. 39, 579 (1992)

14. J. de Rosny, M. Fink, Overcoming the diffraction limit in wave physics using a timereversal mirror and a novel acoustic sink, Phys. Rev. Lett. 89, 124301 (2002)

15. Y. Chong, L. Ge, H. Cao, A.D. Stone, Coherent perfect absorbers: Time-reversed lasers, Phys. Rev. Lett. 105, 053901 (2010)

16. H. Noh, S.M. Popoff, H. Cao, Broadband subwavelength focusing of light using a passive sink, Opt. Exp. 21, 017435 (2013)

17. G. Ma, M. Yang, S. Xiao, Z. Yang, P. Sheng, Acoustic metasurface with hybrid resonances, Nature Mater. 13, 873 (2014)

18. V. Bacot, M. Labousse, A. Eddi, M. Fink, E. Fort, Time reversal and holography with spacetime transformations, Nature Phys. (2016) doi: 10.1038/nphys3810

19. J.T. Mendonça, P.K. Shukla, Time refraction and time reflection: Two basic concepts, Phys. Scripta, 65, 160 (2002)

20. J.T. Mendonça, A.M. Martins, A. Guerreiro, Temporal beam splitter and temporal interference, Phys. Rev. A 68, 043801 (2003)

21. M. Salem, A. Caloz, Space-time cross-mapping and application to wave scattering (2015) arXiv preprint arXiv:1504.02012

Open Access This is an Open Access article distributed under the terms of the Creative Commons Attribution License (http://creativecommons.org/licenses/by/4.0), which permits unrestricted use, distribution, and reproduction in any medium, provided the original work is properly cited. 